\title{
Attributable Length of Stay, Mortality Risk, and Costs of Bacterial Health Care-Associated Infections in Australia: A Retrospective Case-cohort Study
}

\author{
X. J. Lee', A. J. Stewardson ${ }^{2,3}$, L. J. Worth ${ }^{4,5}$, N. Graves ${ }^{6}$, and T. M. Wozniak ${ }^{7,0}$ \\ ${ }^{1}$ Australian Centre for Health Services Innovation, Queensland University of Technology, Queensland, Australia, ${ }^{2}$ Department of Infectious Diseases, The Alfred and Central Clinical School, \\ ${ }^{3}$ Monash University, Victoria, Australia, ${ }^{4}$ Victorian Healthcare Associated Infection Surveillance System Coordinating Centre, Doherty Institute, Victoria, Australia, ${ }^{5}$ National Centre for Infections \\ in Cancer, Sir Peter MacCallum Department of Medicine, University of Melbourne, Victoria, Australia, ${ }^{6}$ Duke University and the National University of Singapore Medical School, Singapore, and \\ ${ }^{7}$ Charles Darwin University, Menzies School of Health Research, Global \& Tropical Health Division, Northern Territory, Australia
}

Background. Unbiased estimates of the health and economic impacts of health care-associated infections (HAIs) are scarce and focus largely on patients with bloodstream infections (BSIs). We sought to estimate the hospital length of stay (LOS), mortality rate, and costs of HAIs and the differential effects on patients with an antimicrobial-resistant infection.

Methods. We conducted a multisite, retrospective case-cohort of all acute-care hospital admissions with a positive culture of 1 of the 5 organisms of interest (Escherichia coli, Klebsiella pneumoniae, Pseudomonas aeruginosa, Staphylococcus aureus, or Enterococcus faecium) from 1 January 2012 through 30 December 2016. Data linkage was used to generate a data set of statewide hospital admissions and pathology data. Patients with bloodstream, urinary, or respiratory tract infections were included in the analysis and matched to a sample of uninfected patients. We used multistate survival models to generate LOS, and logistic regression to derive mortality estimates.

Results. We matched 20390 cases to 75635 uninfected control patients. The overall incidence of infections due to the 5 studied organisms was 116.9 cases per 100000 patient days, with E. coli urinary tract infections (UTIs) contributing the largest proportion (51 cases per 100000 patient days). The impact of a UTI on LOS was moderate across the 5 studied pathogens. Resistance significantly increased LOS for patients with third-generation cephalosporin-resistant K. pneumoniae BSIs (extra 4.6 days) and methicillin-resistant S. aureus BSIs (extra 2.9 days). Consequently, the health-care costs of these infections were higher, compared to corresponding drug-sensitive strains.

Conclusions. The health burden remains highest for BSIs; however, UTIs and respiratory tract infections contributed most to the health-care system expenditure.

Keywords. antimicrobial resistance; length of stay; hospital-associated infections; mortality; cost.

Health care-associated infections (HAIs) are often preventable [1], but continue to pose a significant burden on patients' health and the economy [2]. On any given day, 1 in 18 patients in a European hospital [3] and 1 in 10 Australian inpatients [4] has a HAI. Impacts of HAI include prolonged hospital stays, readmissions, disability, and increased risk of treatment failure [1,5-7]. Antimicrobial resistance (AMR) is a recognized global public health emergency that poses a fundamental threat to human health, development, and security [8].

Received 8 April 2020; editorial decision 7 August 2020; accepted 17 August 2020; published online August 21, 2020.

Correspondence: T. M. Wozniak, Charles Darwin University, Menzies School of Health Research, Level 10, 410 Ann Street, Brisbane Queensland 4000, Australia (Teresa.wozniak@ menzies.edu.au).

\section{Clinical Infectious Diseases ${ }^{\circledR} \quad$ 2020;XX(XX):1-9}

(C) The Author(s) 2020. Published by Oxford University Press for the Infectious Diseases Society of America. This is an Open Access article distributed under the terms of the Creative Commons Attribution-NonCommercial-NoDerivs licence (http://creativecommons.org/licenses/ by-nc-nd/4.0/), which permits non-commercial reproduction and distribution of the work, in any medium, provided the original work is not altered or transformed in any way, and that the work is properly cited. For commercial re-use, please contact journals.permissions@oup.com DOI: 10.1093/cid/ciaa1228
While the burden of HAIs is well described, many studies either do not appropriately adjust for time-dependent bias [9] or focus on bloodstream infections (BSIs) only $[6,10,11]$, omitting other highly prevalent HAIs, such as urinary tract infections (UTIs) [12]. Few have focused on a comprehensive assessment of the AMR burden $[10,11,13]$. Hence, the current evidence base for the impact of HAIs is likely to be overestimated, because of time-dependent bias and its effect on hospital length of stay (LOS) estimates $[14,15]$, or be narrowed in scope. This creates a gap in our ability to assess the health benefits and cost effectiveness that (new) infection control strategies may offer in reducing HAIs and AMR.

The objective of this study was to provide robust estimates of the health burden of 5 bacterial HAIs and the additional impact of AMR, using multistate modeling. We estimated the impacts of Escherichia coli, Klebsiella pneumoniae, Pseudomonas aeruginosa, Staphylococcus aureus, and Enterococcus faecium from multiple sites (BSI, UTI, and respiratory tract infections [RTI]) on patient in-hospital stay and mortality. In addition, the economic cost impact of these infections to the Australian 
health-care system was estimated, including the additional AMR cost impact.

\section{METHODS}

\section{Setting and Study Design}

This was a multi-site, retrospective case-cohort study of all acute-care hospital admissions in Queensland, Australia, from 1 January 2012 through 30 December 2016. Patients with a positive culture of 1 of the 5 organisms of interest, including E. coli, K. pneumoniae, P. aeruginosa, S. aureus, or E. faecium, from multiple sites (BSI, UTI, and RTI) were matched with a sample of uninfected patients. Data linkage was used to generate a data set that matched Queensland Hospital Admitted Patients Data Collection [16] with Queensland Department of Health Pathology [17]. Linkage was done by matching the hospital and hospital-level patient identifiers and by ensuring that sample collection dates fell within recorded admission and discharge dates. Up to 3 uninfected patients were matched based on hospital, age group, sex, and separation date.

\section{Definitions}

Health care-associated (HA)-BSI was defined as a positive blood culture present $>48$ hours after admission. HA-UTI was defined as a patient having a urine culture $>48$ hours after admission with no more than 2 species of organisms identified and a count of $>10^{5}$ colony-forming units of bacteria per milliliter in a urine specimen. HA-RTI was defined as a positive $P$. aeruginosa smear or culture and a count of $>10^{4}$ colonyforming units per milliliter from lung tissue or pleural fluid present $>48$ hours after admission. Only the first positive culture per patient was analyzed. These definitions are consistent with published and accepted criteria for defining HA-BSI [18], HA-UTI [12, 18, 19], and HA-RTI [20].

Antibiotic susceptibility results were provided as European Committee on Antimicrobial Susceptibility Testing interpreted values (resistant, intermediate, and susceptible). All resistant and intermediate results were regarded as "resistant" for the purpose of phenotype analysis. Third-generation cephalosporin (3GC) resistance was inferred from ceftazidime and ceftriaxone resistance, and methicillin resistance in $S$. aureus was inferred from resistance to flucloxacillin.

There were 10 exposure groups:

1. 3GC-resistant K. pneumoniae

2. 3GC-sensitive $K$. pneumoniae

3. 3GC-resistant E. coli

4. 3GC-sensitive E. coli

5. Ceftazidime-resistant $P$. aeruginosa

6. Ceftazidime-sensitive $P$. aeruginosa

7. Methicillin-resistant $S$. aureus (MRSA)

8. Methicillin-sensitive $S$. aureus (MSSA)
9. Vancomycin-resistant E. faecium (VRE)

10. Vancomycin-sensitive E. faecium (VSE)

\section{Statistical Analysis}

A multistate survival model was used to estimate the difference in LOS between those with an infection and those without [21] using the etm-package in R [22] and adapting for the casecohort design [23] of our data. BSIs, UTIs, and RTI for the 5 organisms were the time-varying exposure of interest, and separate analyses were performed for each organism and infection site, referred to subsequently as "infection groupings." The multistate model has 4 states: uninfected, infected, discharged alive, and died in hospital (Figure 1).

Patients entered the model through the "uninfected" state. Transition between states was determined by transition hazards ( $\alpha$ ), accounting for both time-dependent and competing risk natures of the events (Supplementary Material). Long hospitalizations were censored at 90 days to remove any long-stay effects [10]. We estimated the excess LOS attributable to resistance by simulating the estimated extra LOS for resistant and susceptible infections from the respective multistate models as separate gamma distributions, and computing the difference. We used 10000 simulations from each fitted distribution to estimate the excess LOS attributable to resistance.

From logistic regressions, we estimated the effect of HAI on in-hospital death as the death odds ratio (OR), which was appropriate for data sets with small amounts of administrative censoring [24]. The death OR quantifies the association between HAI and hospital mortality risk. Estimates were obtained from 4 regression models, which differed by the level of grouping structure (ie, all $S$. aureus versus MRSA versus $S$. aureus BSI versus MRSA BSI). Death OR estimates of resistant and susceptible

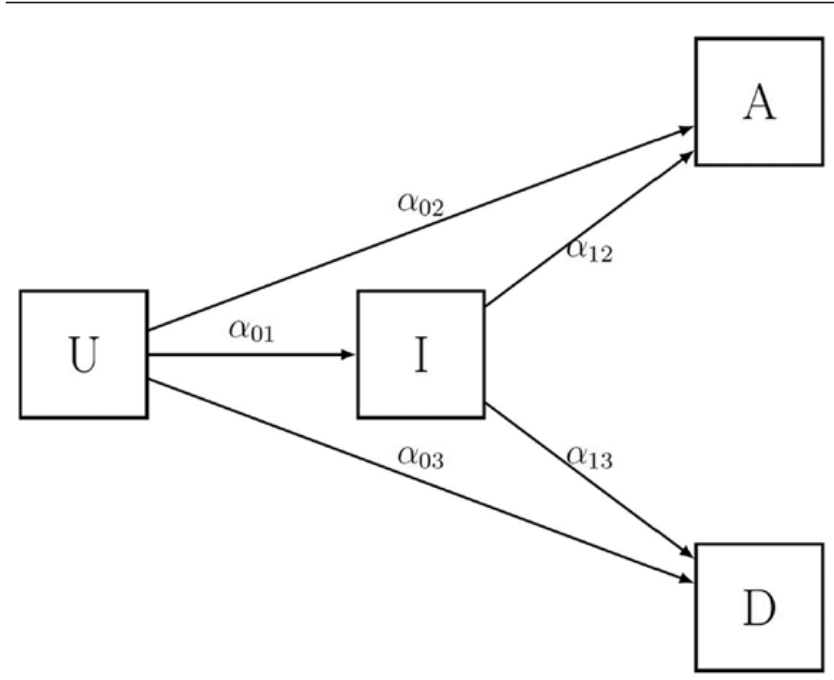

Figure 1. Multi-state model used to determine the length of stay. The alphas $(\alpha)$ represent the transition hazards. Abbreviations: A, patients discharged alive; D, patients who died in hospital; I, infected patients; U, uninfected patients. 
pairings were compared using the estimated marginal means (Supplementary Material, logistical regression). Results are presented as ORs and 95\% confidence intervals (CIs). We included potential confounding variables of age, sex, admission year, time from admission to infection, hospital peer-group and remoteness [25], and comorbidities as additional covariates in the logistic regression model. Comorbidities were identified using a validated algorithm based on International Classification of Disease, 10th Revision, Australian Modification (ICD-10-AM) coding [26] and were converted to the Charlson comorbidity index [27].

\section{Cost of Infection}

The cost of infection was calculated as a product of the prolongation of LOS associated with infection and a monetary value of a bed day. First, we used an estimate per bed day (\$250.40 Australian dollars) [28] reported from a contingent valuation study that elicited a hospital Chief Executive Officer's "willingness to pay" to release a bed day from some infection-reducing intervention. This is akin to the opportunity costs (and referred to hereafter as "opportunity costs") of a bed day, and provides a valuation more likely to be used for decision-making under scarce resources. Second, we used the "accounting costs" per bed days, obtained by dividing the total annual hospital budget by the number of bed days supplied during the same period (\$2721.8 Australian dollars, estimated by adjusting 2016-17 estimates to 2020 prices) [29]. Cost of resistance was calculated as the additional costs of a resistant infection over the corresponding susceptible infection pairing. Annual costs for the infection groups were calculated by multiplying the cost per infection and the incidence density for each group. Results are presented as the average annual costs over the 5-year period of the data set, with $95 \%$ CIs. All costs were converted to 2020 US dollars (USD) using the XE Currency Converter, Live Rates [30].

\section{Ethics}

Ethical approval, including consent waiver, was granted by the Queensland University of Technology Human Research Ethics Committee (HREC1700000232).

\section{RESULTS}

Overall, data for 96025 patients from 134 Queensland hospitals were analyzed. Studied hospitals included facilities located in major cities [31] $(n=18)$, regional hospitals $(n=74)$, and rural health-care services $(n=42)$, with the majority of patient data collected from hospitals in major cities (62.3\%). Evaluable data were comprised of 20390 infected cases with 1 of the 5 organisms of interest, and 75635 uninfected controls. Patients with UTIs were older than those with BSIs, and females were overrepresented, except for in the $S$. aureus UTI group, where the proportion of males was high (72\%). The average age of the control group was 68 years old (standard deviation [SD], 23), whilst patients classified as cases had a similar overall age of 68 years old (SD, 20), but a smaller proportion of females $(59 \%$ in cases versus $66 \%$ in controls; see Table 1 ).

The overall incidence of the 5 studied HAIs was 116.8 per 100000 patient days (Table 1). More HAIs were due to Gramnegative bacteria than to Gram-positive bacteria. UTIs secondary to E. coli were most common, with an incidence of 51.5 cases per 100000 patient days, whilst BSIs were most frequently due to $S$. aureus (5.7 cases per 100000 patient days). The incidence of $P$. aeruginosa RTI was 11.6 cases per 100000 patient days. Drug-sensitive strains were more prevalent than drugresistant strains (Table 1 ).

All-cause in-hospital mortality was highest in patients with a BSI, compared those with a UTI or RTI. Enterococcus faecium BSIs were associated with the greatest number of deaths, especially in those who were infected with a resistant strain $(25.6 \%$, $\mathrm{n}=43$ ). The all-cause mortality proportions for patients with E. coli and P. aeruginosa BSIs were $16.7 \%$ and $15.8 \%$ of patients, respectively.

\section{Infection-attributable Excess Hospital Length of Stay}

HA-BSIs resulted in a greater excess LOS in hospital than UTIs or RTIs (Figure 2). Staphylococcus aureus BSIs resulted in an additional 5.3 days (95\% CI, 5.2-5.5) in hospital, followed by $P$. aeruginosa and E. faecium at 3.5 days (95\% CI, 3.3-3.7) and 3.4 days (95\% CI, 3.2-3.6), respectively (Figure 2A). Patients with a MRSA BSI stayed in hospital for an additional 7.9 days (95\% CI, 7.8-8.1), which was 2.9 days (95\% CI, $2.7-$ 3.1) longer than MSSA BSI patients. Similarly, patients with a 3GC-resistant $K$. pneumoniae BSI stayed in hospital for an additional 7.3 days (95\% CI, 7.1-7.4), whilst those with drugsensitive K. pneumoniae had an excess LOS of 2.7 days, resulting in an additional 4.6 days (95\% CI, 4.3-4.8) attributable to resistance. There was no additional impact on hospital LOS for patients who had a E. coli infection, either drug-sensitive or drug-resistant (Figure 2). Patients with a vancomycin-sensitive strain of E. faecium BSI stayed in hospital an additional 2.5 days (95\% CI, 2.1-2.9) compared with patients with VRE. The same effect was not seen in patients with a urinary E. faecium infection (Figure $2 B$ ).

Across all 5 infections, the impact of a UTI on hospital LOS was moderate (Figure $2 B$ ). The additional impact on hospital LOS for patients with a drug-resistant infection was more notable in patients with a 3GC-resistant K. pneumoniae UTI, who stayed in hospital an additional 2 days (95\% CI, 1.7-2.2) compared to patients infected with a drug-sensitive strain.

Patients with a $P$. aeruginosa RTI stayed in hospital for an additional 2.8 days (95\% CI, 2.7-3.0) compared to uninfected controls (Figure 2C). Ceftazidime-resistant infections resulted in a LOS of 1.5 days, whilst patients with a ceftazidime-sensitive infection stayed in hospital for an estimated 3 days (95\% CI, 2.9-3.2). 
Table 1. Patient Characteristics for the Study Population, Queensland, 2012-2016

\begin{tabular}{|c|c|c|c|c|c|c|}
\hline & Number & Age (SD) & Female, \% & Died in hospital, \% & LOS, median (IQR) & Incidence/100 000 \\
\hline Controls, uninfected & 75635 & $67.7(23.2)$ & 65.6 & 5.7 & $4(3-8)$ & \\
\hline Cases, infected & 20390 & $67.6(20.3)$ & 59.4 & 7.2 & $21(11-38)$ & 116.88 \\
\hline S. aureus BSIs & 999 & $59.9(23.1)$ & 32.1 & 13.3 & $25(16-43)$ & 5.73 \\
\hline MRSA BSIs & 140 & $56(23.2)$ & 35.7 & 12.9 & $34(20-56)$ & .80 \\
\hline MSSA BSIS & 859 & $60.5(23)$ & 31.5 & 13.4 & $24(15-41)$ & 4.92 \\
\hline S. aureus UTIs & 497 & $67.3(21)$ & 28.2 & 8.2 & $21(11-36)$ & 2.85 \\
\hline MRSA UTIS & 81 & $71.2(17.4)$ & 28.4 & 9.9 & $33(17-49)$ & .46 \\
\hline MSSA UTIS & 416 & $66.5(21.5)$ & 28.1 & 7.9 & $19(11-33)$ & 2.38 \\
\hline E. faecium BSIs & 107 & 57.7 (18.3) & 36.4 & 22.4 & $35(20-53)$ & .61 \\
\hline VRE BSIs & 43 & $60.9(14.9)$ & 37.2 & 25.6 & $31(21-49)$ & .25 \\
\hline VSE BSIs & 64 & $55.5(20)$ & 35.9 & 20.3 & $36(20-55)$ & .37 \\
\hline E. faecium UTIs & 727 & $70.3(17.1)$ & 67.1 & 8.7 & $24(14-41)$ & 4.17 \\
\hline VRE UTIS & 323 & $70.6(17.1)$ & 65.6 & 7.7 & $27(15-42)$ & 1.85 \\
\hline VSE UTIS & 404 & $70.1(17.1)$ & 68.3 & 9.4 & $22(12-40)$ & 2.32 \\
\hline E. coli BSIs & 588 & $62.2(21.3)$ & 39.3 & 16.7 & $22(14-36)$ & 3.37 \\
\hline REC BSIs & 45 & $61.3(20.6)$ & 31.1 & 15.6 & $24(17-36)$ & .26 \\
\hline SEC BSIS & 543 & $62.2(21.4)$ & 40 & 16.8 & $22(13-36)$ & 3.11 \\
\hline E. coli UTIs & 8952 & $69.2(19.7)$ & 75.4 & 4.9 & $18(10-35)$ & 51.32 \\
\hline REC UTIS & 448 & $68.4(20.1)$ & 65.6 & 3.3 & $19(10-37)$ & 2.57 \\
\hline SEC UTIS & 8504 & $69.3(19.7)$ & 75.9 & 5 & $18(10-35)$ & 48.75 \\
\hline K. pneumoniae BSIs & 354 & $58.1(20.8)$ & 35 & 15.5 & $27(16-45)$ & 2.03 \\
\hline RKP BSIs & 14 & $53.4(17.2)$ & 28.6 & 14.3 & $24(22-40)$ & .08 \\
\hline SKP BSIS & 340 & $58.3(20.9)$ & 35.3 & 15.6 & $27(16-45)$ & 1.95 \\
\hline K. pneumoniae UTIs & 2420 & $66.2(20.4)$ & 59.6 & 6.7 & $21(11-37)$ & 13.87 \\
\hline RKP UTIS & 120 & $66.2(18.8)$ & 56.7 & 5.8 & $24(14-46)$ & .69 \\
\hline SKP UTIS & 2300 & $66.2(20.5)$ & 59.8 & 6.7 & $21(11-37)$ & 13.18 \\
\hline P. aeruginosa BSIs & 285 & $61.4(20.3)$ & 31.2 & 15.8 & $28(17-45)$ & 1.63 \\
\hline RPA BSIs & 23 & $54(24.6)$ & 34.8 & 17.4 & $34(24-64)$ & .13 \\
\hline SPA BSIS & 262 & $62.1(19.8)$ & 30.9 & 15.6 & $26(17-45)$ & 1.50 \\
\hline P. aeruginosa UTIs & 3519 & $71.7(18.5)$ & 48.4 & 5.9 & $24(12-43)$ & 20.17 \\
\hline RPA UTIS & 299 & $68.3(19.5)$ & 43.8 & 8 & $31(18-52)$ & 1.71 \\
\hline SPA UTIS & 3220 & $72(18.4)$ & 48.9 & 5.7 & $23(12-42)$ & 18.46 \\
\hline$P$. aeruginosa respiratory & 1942 & 62 (21.6) & 40 & 10.6 & $21(12-38)$ & 11.13 \\
\hline RPA respiratory infections & 338 & $60(21.9)$ & 39.6 & 11.5 & $20(12-43)$ & 1.94 \\
\hline SPA respiratory infections & 1604 & $62.4(21.5)$ & 40.1 & 10.4 & $21(12-38)$ & 9.19 \\
\hline
\end{tabular}

Abbreviations: BSI, bloodstream infection; E. coli, Escherichia coli; E. faecium, Enterococcus faecium; IQR, interquartile range; K. pneumoniae, Klebsiella pneumoniae; LOS, length of stay; MRSA, methicillin-resistant S. aureus; MSSA, methicillin-sensitive S. aureus; P. aeruginosa, Pseudomonas aeruginosa; RPA, resistant $P$. aeruginosa; REC, resistant E. coli; RKP, resistant K. pneumoniae; S. aureus, Staphylococcus aureus; SD, standard deviation; SEC, sensitive E. coli; SKP, sensitive K. pneumoniae; SPA, sensitive P. aeruginosa; UTI, urinary tract infection; VRE, vancomycin-resistant $E$. faecium; VSE, vancomycin-sensitive $E$. faecium.

\section{Infection-attributable Mortality}

All BSIs were associated with increased odds of death (Figure $3 A$ ). Enterococcus faecium BSI patients were associated with a death OR of 4.5 (95\% CI, 2.7-7.3) compared with uninfected patients. This was followed by K. pneumoniae BSI (OR, 3.1; 95\% CI, 2.3-4.3) and E. coli BSI (OR, 2.9; 95\% CI, 2.3-3.7) patients, who had an additional 3-fold increased risk of death compared to control patients. The death OR was significant only for E. faecium UTI patients (OR, 1.4; 95\% CI, 1.1-1.8; Figure $3 B$ ) and $P$. aeruginosa RTI patients (OR, 2.4; 95\% CI, 2.0-2.8; Figure 3C). Differences in death ORs between resistant and susceptible infections were not statistically significant, but were most notable in P. aeruginosa (BSI OR, 1.6 [95\% CI, -1.1 to 4.2]; UTI OR, 1.6 [95\% CI, .5-2.7]; and RTI OR, 1.6 [95\% CI, .6-2.1]).

\section{Infection-attributable Cost}

Staphylococcus aureus BSIs were associated with the highest cost. Opportunity costs were estimated as USD \$909.1 (SD, \$97.6) per infection, and hospital accounting costs were USD \$9877.8 (SD, \$999.8; Table 2).

Hospitalization of patients with a resistant infection was associated with higher costs than patients with a drug-sensitive strain across the 5 infections, except for patients with VRE bacteremia and ceftazidime-resistant $P$. aeruginosa RTI (Table 2). Opportunity costs attributable to hospital LOS were lower in VRE BSI patients (USD \$318) compared with VSE BSI patients (USD \$760) and in patients with a P. aeruginosa RTI (Table 2). For all remaining infections, resistance-attributable costs were higher or comparable to those in patients with drug-sensitive infections. We estimate an additional USD \$753.5 (SD, 147.9) 
A. Bloodstream infections

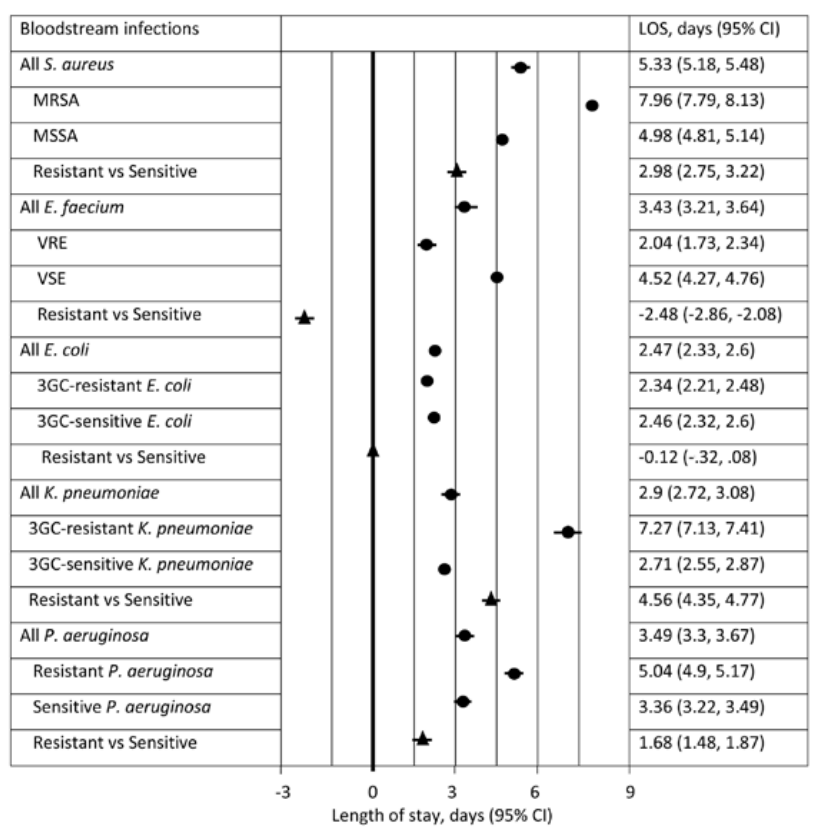

B. Urinary tract infections

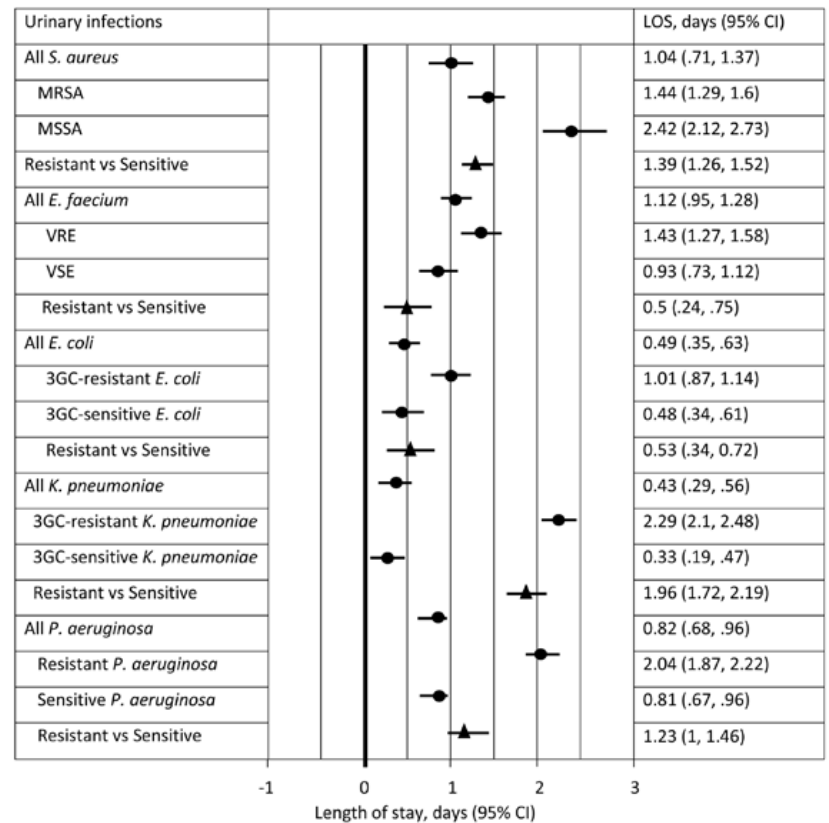

C. Respiratory infections

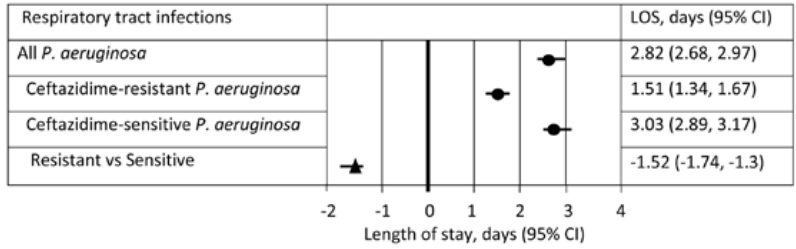

Figure 2. Infection-attributable LOS for health care-associated infections in the $(A)$ bloodstream, $(B)$ urinary tract, and $(C)$ respiratory tract, Queensland, 2012-2016. Abbreviations: 3GC, third-generation cephalosporin; Cl, confidence interval; E. coli, Escherichia coli; E. faecium, Enterococcus faecium; K. pneumoniae, Klebsiella pneumoniae; LOS, length of stay; MRSA, methicillin-resistant $S$. aureus; MSSA, methicillin-sensitive $S$. aureus; P. aeruginosa, Pseudomonas aeruginosa; S. aureus, Staphylococcus aureus; VRE, vancomycin-resistant $E$. faecium; VSE, vancomycin-sensitive $E$. faecium.

per 3GC-resistant K. pneumoniae BSI; USD \$503.2 (SD, 172.9) per MRSA BSI; and USD \$342.6 (SD, 123.1) per ceftazidimeresistant $P$. aeruginosa BSI. UTIs were associated with increased bed-day costs for all organisms, but the increases were notably smaller than the corresponding BSI costs. The accounting costs for each of the organisms are presented in Table 2.

Averaged over the 5-year study period, the annual costs estimated using accounting estimates of bed-day costs and incidences were highest for P. aeruginosa RTI (USD \$2 056 492; 95\% CI, \$1 658 763-2 489 062; Figure 4C) and S. aureus BSI (USD \$1 973 594; 95\% CI, \$1 600 910-2 375 414; Figure 4A), compared to uninfected patients. This was followed by E. coli UTI, with an estimated annual opportunity cost of USD\$ 1699756 per year (95\% CI, \$1 191 226-2 323 857; Figure 4B) that was over 3-fold higher than the corresponding BSI cost (USD \$510 507; 95\% CI, \$408 993-622 654; Figure 4A). In contrast, S. aureus BSI costs (USD $\$ 1973$ 594) were 7 times the annual S. aureus UTI costs (USD $\$ 277655$; Figure $4 B$ ). For the 5 organisms, the annual HA-UTI cost was USD \$3.7 million and the annual HA-BSI cost was USD \$3.4 million. The annual cost of HA-RTI, which includes only $P$. aeruginosa, was USD \$2.1 million (Figure $4 C$ ). The combined accounting costs of patients with BSI, UTI, and RTI drug-sensitive infections were higher than the corresponding drug-resistant costs, and amounted to USD \$7.8 million per year (USD \$7 762 293) and USD \$1.6 million per year (USD \$1 614 496), respectively.

\section{DISCUSSION}

We provide a comprehensive estimate of the impact of antibiotic-sensitive and -resistant bacterial HAIs in Australia. Our study expands upon the literature by more fully adjusting estimates of morbidity (excess LOS) and mortality in patients with drug-resistant UTIs and $P$. aeruginosa RTI. We used data linkage to identify 20469 patients infected with 1 of 5 clinically significant organisms; of these, 1693 patients' infections were due to antibiotic-resistant bacteria. The combined (BSI, UTI, and RTI) antibiotic-resistant infections accounted for 717 extra days of hospital stay annually; suggested total annual costs were 
A. Bloodstream infections

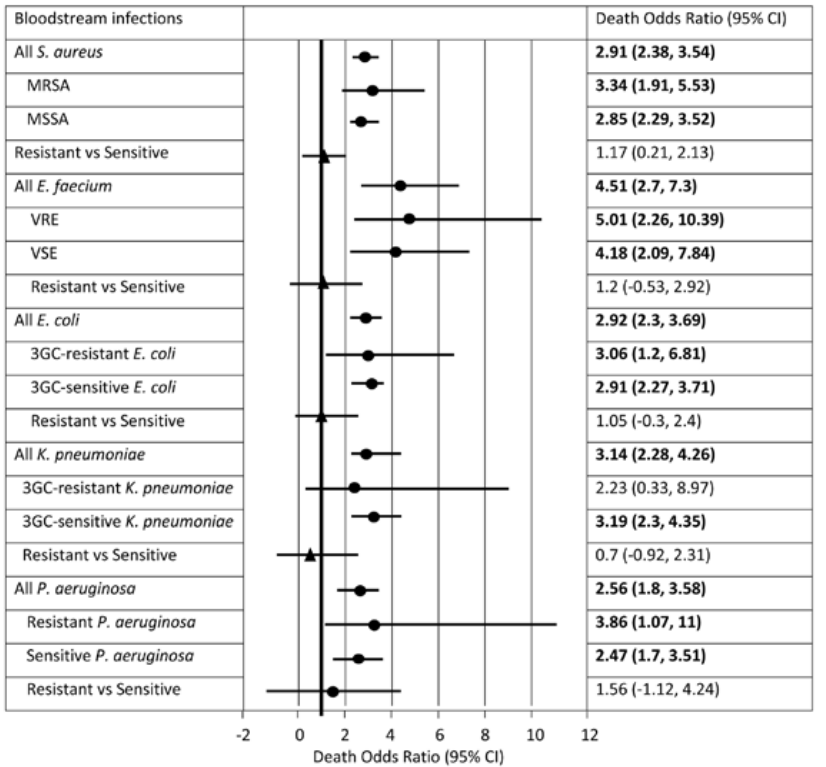

C. Respiratory infection

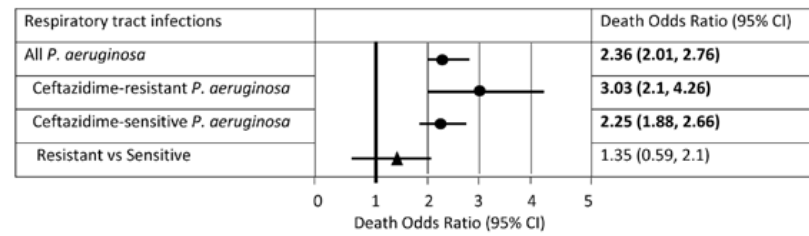

B. Urinary infections

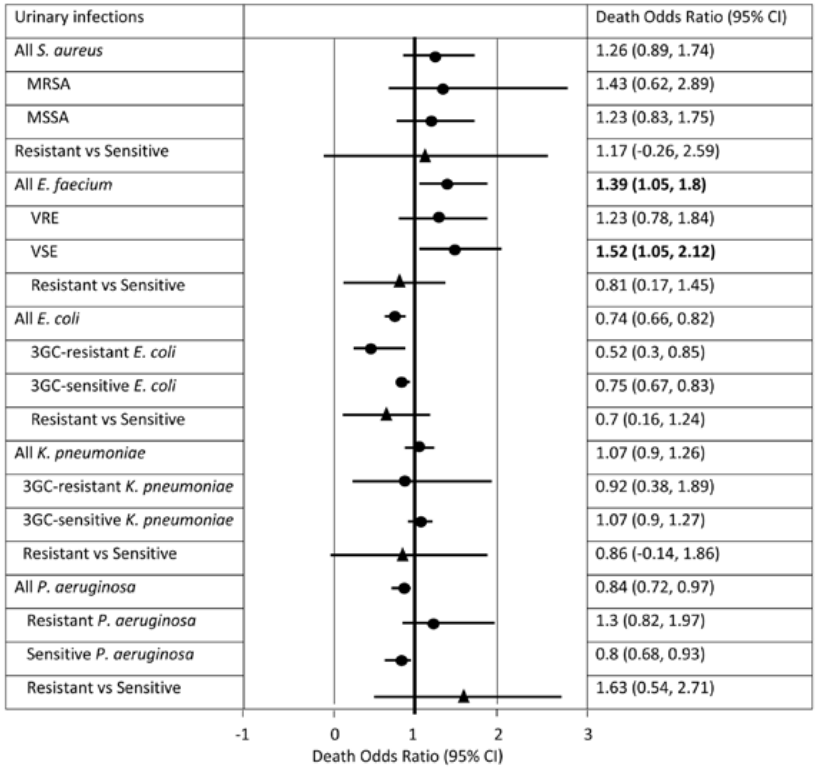

Figure 3. Infection-attributable mortality for patients with health care-associated infections in the $(A)$ bloodstream, $(B)$ urinary tract, and $(C)$ respiratory tract, Oueensland, 2012-2016. Odds ratios presented were for the reference category: that is, an uninfected female patient with age equal to the mean age in the cohort who was admitted in 2012 to a Principal Referral Hospital located in a metropolitan setting and had no comorbidities. For the resistant versus susceptible comparison, the time to infection covariate was taken to be the average time to infection of infected patients in the data set. Dots represent the point estimate. Triangles represent the change between Resistant and Sensitive groups. Bold indicates significant results where the odds ratio was above 1. Abbreviations: 3GC, third-generation cephalosporin; Cl, confidence interval; E. coli, Escherichia coli; E. faecium, Enterococcus faecium; K. pneumoniae, Klebsiella pneumoniae; MRSA, methicillin-resistant S. aureus; MSSA, methicillin-sensitive S. aureus; P. aeruginosa, Pseudomonas aeruginosa; S. aureus, Staphylococcus aureus; VRE, vancomycin-resistant E. faecium; VSE, vancomycin-sensitive E. faecium.

USD \$130 297 when opportunity cost estimates for bed days were used, and USD \$1.6 million when accounting costs were used. Our results are comparable with the limited available international data $[10,32-34]$, indicating that both resistant and susceptible bacterial infections cause substantial health and economic burdens. Susceptible infections were more prevalent than resistant infections, and therefore contributed proportionally to health and economic burden.

Current estimates of the resistance-attributable morbidity are available for Enterobacteriaceae [10]; P. aeruginosa [35], E. faecium [36], and S. aureus [9, 10, 35] BSIs; and RTIs [35] caused by $P$. aeruginosa and $S$. aureus. Adjusting for patient age, sex, and admission year, we demonstrated that resistance was an important contributor to increasing hospital LOS. Patients with 3GC-resistant K. pneumoniae BSIs and MRSA BSIs stayed in hospital for an additional 4.4 days and 2.9 days, respectively, when compared to patients with drug-sensitive infections. This is comparable to other reported fully-adjusted LOS estimates for K. pneumoniae (4.9 days) and MRSA (2.5 days) [10]. We provide the first report of fully adjusted estimates of morbidity and mortality for $P$. aeruginosa infections, as previous studies used methods that partially adjusted for time-dependent bias [35]. We show that patients infected with ceftazidime-resistant $P$. aeruginosa BSIs stayed in hospital an extra 1.7 days and had a higher death OR (OR, 3.9 versus 2.5 , respectively), compared to patients with a drug-sensitive strain. Interestingly, the hospital LOS in patients with a ceftazidime-resistant $P$. aeruginosa RTI was shorter than that in patients with a drug-sensitive strain, which may be attributable to the competing influence of higher odds of death in this patient population.

Infections acquired in a hospital, whether they are resistant or sensitive, increase the LOS, and more than likely also impact the disease severity and likelihood of survival. However, quantifying the LOS in hospital and any changes to the risk of mortality in these patients is challenging, because the exposure (infection) is a time-dependent variable. Hence, the longer a patient stays in a hospital, the higher their risk of acquiring an infection. Modeling the infection time during a patient's hospital 


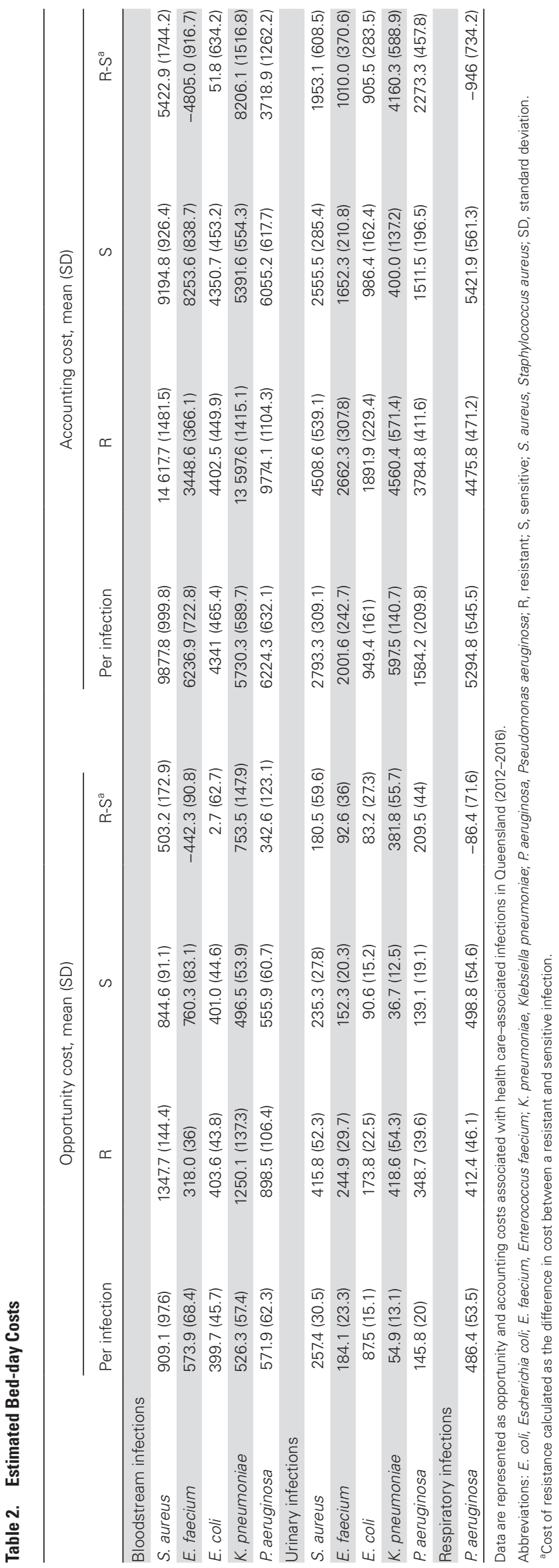

admission using multistate statistical models [37] appropriately adjusts for time-dependent bias in such studies and accounts for the competing risk nature of the events of interest. In-hospital mortality is such an event of interest, in that a higher mortality risk will lead to a reduced LOS and reduced costs associated with the LOS. Hence, the patient's discharge and death risk affect the patient's LOS and infection risk. We found that patients with VRE bacteremia had higher odds of death (OR, 5), compared to VSE BSI (OR, 4.2), as well as shorter hospital LOS (2 days versus 4.5 days, respectively). As a retrospective, observational study, we can't exclude the possibility of residual confounding, which may in part explain the excess LOS associated with VRE BSIs compared with VSE BSIs. However, the higher odds of dying may offset the costs incurred by patients' hospital LOS, and we found a lower per-infection cost for VRE BSIs (USD $\$ 318$ ), compared with VSE BSIs (USD $\$ 760$ per infection). The flow-on effect from that may be that the lower health-care costs associated with resistant infections may undermine efforts in targeting AMR as a priority. This highlights that overall LOS costs are not sufficient indicators alone for program evaluations and priority settings, and should always be supported by estimates of morbidity and mortality associated with HAIs and AMR. In addition to the direct costs estimated in this study, modeling the unique attributes of value-such as the indirect benefits that accrue from preventing the transmission of AMR and the protective effects of existing antibiotics-will be critical in future studies [38].

Our economic burden estimates focused on the acute cost of infections, specifically due to prolongation of a patient's LOS, as valued by either Australian hospital Chief Executive Officers or accounting costs. While these are conservative estimates of the economic burden of infections, they provide information to support local and international hospital decision-makers in planning for future infection control and prevention programs. It is possible to include the cost associated with a change in mortality risk observed by costing the life-years lost due to in-hospital death (please see Supplementary Material). However, these cost estimates should be interpreted and used cautiously, as it is possible that there are other contributing and confounding factors that were not included in the analysis mortality risk. An alternative approach would be to estimate the disability-adjusted life-years attributable to infections and the associated costs [6].

\section{Limitations}

The 5 infections included in the analysis were selected due to a high burden of disease [13,39]; a relative paucity of estimates of the infection burden, especially for noninvasive sites [40]; and a growing concern about antibiotic resistance [41, 42]. We did not focus upon rare pathogens, such as carbapenemaseproducing Enterobacteriaceae [43], which, although likely to result in substantial morbidity and mortality, have not yet resulted in a burden at a national level. Some infection groups 
A. Bloodstream infections

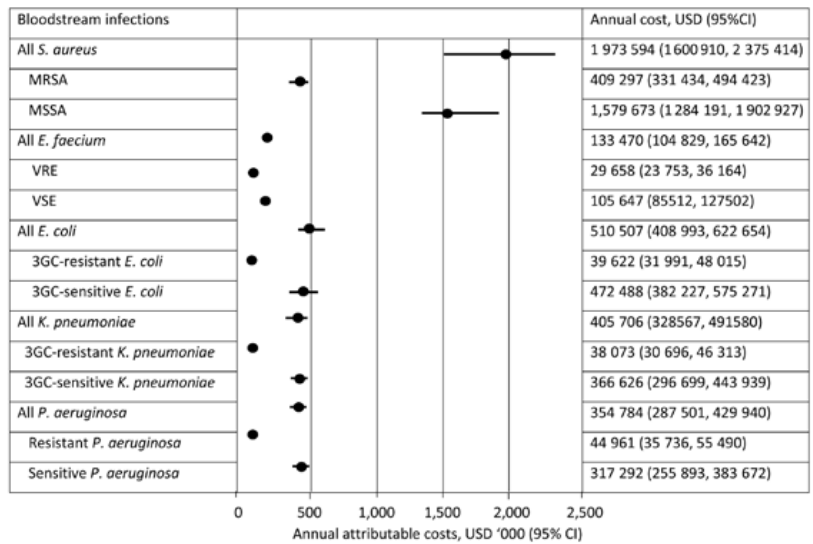

C. Respiratory infections

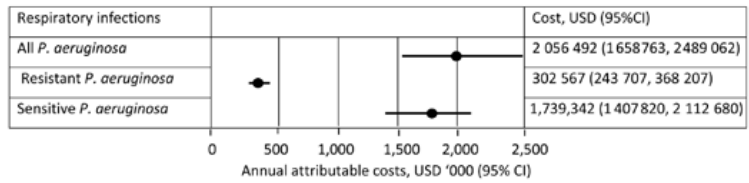

B. Urinary infections

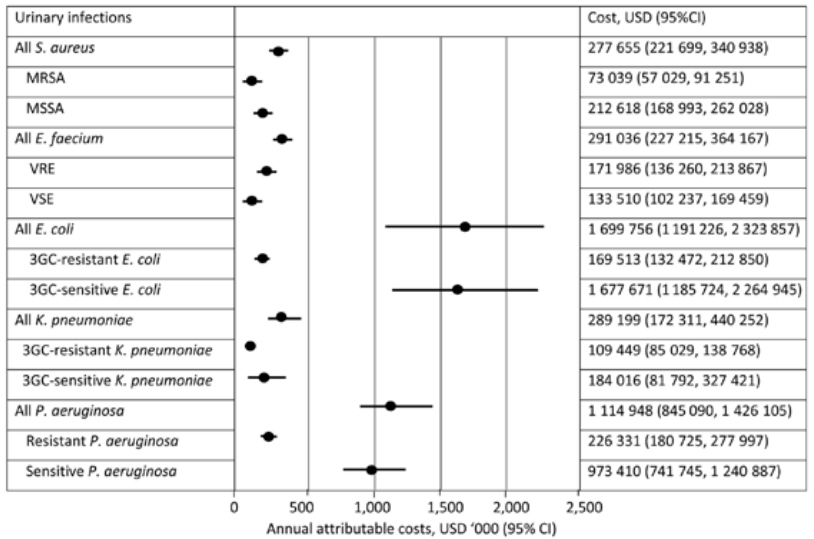

Figure 4. Infection-attributable annual costs for health care-associated infections in the $(A)$ bloodstream, $(B)$ urinary tract, and $(C)$ respiratory tract, Queensland, 20122016. Abbreviations: 3GC, third-generation cephalosporin; Cl, confidence interval; E. coli, Escherichia coli; E. faecium, Enterococcus faecium; K. pneumoniae, Klebsiella pneumoniae; MRSA, methicillin-resistant S. aureus; MSSA, methicillin-sensitive S. aureus; P. aeruginosa, Pseudomonas aeruginosa; S. aureus, Staphylococcus aureus; USD, United States dollar; VRE, vancomycin-resistant E. faecium; VSE, vancomycin-sensitive E. faecium.

(ie, MRSA BSI) have small numbers, which is consistent with annual Australian summaries [44], and are likely due to the exclusion of community-associated infections and effective infection and control practices in Australian hospitals. In the absence of more detailed clinical information, we were not able to confirm the presence of an active infection for patients with urinary or respiratory tract pathogens. Hence, the current study may suffer from misclassification bias by assuming that the microbiological criteria for a positive culture that includes density of bacteria equates to an infection. Additionally, we did not have information on severity of illness (ie, The Acute Physiology and Chronic Health Evaluation or Pitt score) or admission to intensive care prior to infection. Although we adjusted for comorbidities using Charlson comorbidities, the severity of illness prior to the onset of infection is a potential confounder in this study. Our data set included cases and matched noninfected patients, where the matching procedure was performed by an external party and was contingent upon available data. We analyzed the data set using a case-cohort approach with appropriate data processing, due to limitations with data extraction (see Supplementary Material). Potential pitfalls of the external matching process include the fact that infected cases may have been sicker than matched controls prior to infection onset, and this may have contributed to the observed extra LOS and increased risk of death. However, adjusting for the timing of infection is more important than adjusting for baseline comorbidities when estimating LOS due to infection, and this is the method we employed [14]. As such, the cost estimates provided are likely to be an underestimate of the true economic burden of HAIs. This is due to the challenge of attributing the change in mortality observed in the data solely to HAI, which is a likely oversimplification of the hospitalized patients' conditions, particularly for those with only a UTI.

We demonstrate the health and economic burdens attributable to a range of antibiotic-susceptible and -resistant HAIs in Australian health-care facilities. The health burden remains highest for BSIs; however, UTIs and RTIs contributed most to the health-care system expenditure.

\section{Supplementary Data}

Supplementary materials are available at Clinical Infectious Diseases online. Consisting of data provided by the authors to benefit the reader, the posted materials are not copyedited and are the sole responsibility of the authors, so questions or comments should be addressed to the corresponding author.

\section{Notes}

Financial support. This work was supported by the Australian National Health and Medical Research Council (NHMRC). T.M.W. is supported by NHMRC "Improving Health Outcomes in the Tropical North: A multidisciplinary collaboration (HOT NORTH)" (grant number GNT1131932) and the Australian Partnership for Preparedness Research on Infectious Disease Emergencies (APPRISE) (grant number GNT1116530) Fellowship. A.J.S. is supported by an NHMRC Early Career Fellowship (grant number GNT1141398).

Potential conflicts of interest. All authors report no potential conflicts. All authors have submitted the ICMJE Form for Disclosure of Potential Conflicts of Interest. Conflicts that the editors consider relevant to the content of the manuscript have been disclosed. 


\section{References}

1. Schreiber PW, Sax H, Wolfensberger A, Clack L, Kuster SP; Swissnoso. The preventable proportion of healthcare-associated infections 2005-2016: systematic review and meta-analysis. Infect Control Hosp Epidemiol 2018; 39:1277-95.

2. World Health Organization. Report on the burden of endemic health careassociated infection worldwide. Geneva, Switzerland: World Health Organization, 2011.

3. European Centre for Disease Prevention and Control. Point prevalence survey of healthcare-associated infections and antimicrobial use in European acute care hospitals. Stockholm, Sweden: European Centre for Disease Prevention and Control, 2013

4. Russo PL, Stewardson AJ, Cheng AC, Bucknall T, Mitchell BG. The prevalence of healthcare associated infections among adult inpatients at nineteen large Australian acute-care public hospitals: a point prevalence survey. Antimicrob Resist Infect Control 2019; 8:114-24.

5. Gandra S, Barter DM, Laxminarayan R. Economic burden of antibiotic resistance: how much do we really know? Clin Microbiol Infect 2014; 20:973-80.

6. Cassini A, Högberg LD, Plachouras D, et al; Burden of Antimicrobial Resistance (AMR) Collaborative Group. Attributable deaths and disability-adjusted lifeyears caused by infections with antibiotic-resistant bacteria in the EU and the European economic area in 2015: a population-level modelling analysis. Lancet Infect Dis 2019; 19:56-66.

7. Burnham JP, Kwon JH, Olsen MA, Babcock HM, Kollef MH. Readmissions with multidrug-resistant infection in patients with prior multidrug resistant infection. Infect Control Hosp Epidemiol 2018; 39:12-9.

8. World Health Organization. News release. High-level meeting on antimicrobial resistance: general assembly of the United Nations. 2016. Available at: https:/ www.un.org/pga/71/2016/09/21/press-release-hl-meeting-on-antimicrobialresistance/. Accessed December 2019.

9. Wozniak TM, Barnsbee L, Lee XJ, Pacella RE. Using the best available data to estimate the cost of antimicrobial resistance: a systematic review. Antimicrob Resist Infect Control 2019; 8:26-38.

10. Stewardson AJ, Allignol A, Beyersmann J, et al. The health and economic burden of bloodstream infections caused by antimicrobial-susceptible and nonsusceptible Enterobacteriaceae and Staphylococcus aureus in European hospitals, 2010 and 2011: a multicentre retrospective cohort study. Euro Surveillance 2016 21:30319-31.

11. Stewardson A, Fankhauser C, De Angelis G, et al. Burden of bloodstream infection caused by extended-spectrum $\beta$-lactamase-producing Enterobacteriaceae determined using multistate modeling at a Swiss University Hospital and a nationwide predictive model. Infect Control Hosp Epidemiol 2013; 34:133-43.

12. Mitchell BG, Ferguson JK, Anderson M, Sear J, Barnett A. Length of stay and mortality associated with healthcare-associated urinary tract infections: a multistate model. J Hosp Infect 2016; 93:92-9.

13. Wozniak TM, Bailey EJ, Graves N. Health and economic burden of antimicrobialresistant infections in Australian hospitals: a population-based model. Infect Control Hosp Epidemiol 2019; 40:320-7.

14. Beyersmann J, Kneib T, Schumacher M, Gastmeier P. Nosocomial infection, length of stay, and time-dependent bias. Infect Control Hosp Epidemiol 2009; 30:273-6.

15. Barnett AG, Beyersmann J, Allignol A, Rosenthal VD, Graves N, Wolkewitz M The time-dependent bias and its effect on extra length of stay due to nosocomial infection. Value Health 2011; 14:381-6.

16. Queensland Department of Health. Queensland hospital admitted patient data collection. Available at: https://www.health.qld.gov.au/hsu/collections/qhapdc. Accessed December 2018

17. Queensland Government. Open data portal. antibiotic susceptibility from OrgTrx. Available at: https://www.data.qld.gov.au/dataset/antibiotic-susceptibility-from-orgtrx. Accessed December 2018.

18. Centers for Disease Control Prevention. National healthcare safety network (NHSN) patient safety component manual. 2018. Available at: https://www.cdc gov/nhsn/pdfs/pscmanual/pcsmanual_current.pdf. Accessed December 2018.

19. Wilson ML, Gaido L. Laboratory diagnosis of urinary tract infections in adult patients. Clin Infect Dis 2004; 38:1150-8.

20. Campbell S, Forbes BA. The clinical microbiology laboratory in the diagnosis of lower respiratory tract infections. J Clin Microbiol 2011;49:S30-3.

21. Barnett AG, Page K, Campbell M, et al. The increased risks of death and extra lengths of hospital and ICU stay from hospital-acquired bloodstream infections: a case-control study. BMJ Open 2013; 3:e003587.

22. Allignol A, Schumacher M, Beyersmann J. Empirical transition matrix of multistate models: the etm package. J Stat Softw 2011; 38:15. doi:10.18637/jss.v038.i04
23. von Cube M, Schumacher M, Palomar-Martinez M, Olaechea-Astigarraga $P$, Alvarez-Lerma F, Wolkewitz M. A case-cohort approach for multi-state models in hospital epidemiology. Stat Med 2017; 36:481-95.

24. Poguntke I, Schumacher M, Beyersmann J, Wolkewitz M. Simulation shows undesirable results for competing risks analysis with time-dependent covariates for clinical outcomes. BMC Med Res Methodol 2018; 18:79-89.

25. Australian Institute of Health and Welfare. Australian hospital peer groups. Health services series no. 66. Cat. no. HSE 170. Canberra, Australia: Australian Institute of Health and Welfare, 2015.

26. Quan H, Sundararajan V, Halfon P, et al. Coding algorithms for defining comorbidities in ICD-9-CM and ICD-10 administrative data. Med Care 2005; 43:1130-9.

27. Gasparini A. Comorbidity: an R package for computing comorbidity scores. J Open Source Softw 2018; 3:648-51.

28. Page K, Barnett AG, Graves N. What is a hospital bed day worth? A contingent valuation study of hospital Chief Executive Officers. BMC Health Serv Res 2017 17:137-45.

29. Australian Institute of Health and Welfare. Hospital resources 2016-17 Australian hospital statistics. Health services series no. 86. Cat. no. HSE 205. Canberra, Australia: Australian Institute of Health and Welfare, 2018. Available at: https://www.aihw.gov.au/getmedia/c5fd554a-3356-474a-808a-5aab43d24708/ aihw-hse-205.pdf.aspx?inline $=$ true

30. XE.com. XE currency converter-live rates. 2020. Available at: https://www. xe.com/currencyconverter/convert $/$ ? Amount $=1 \&$ From $=U S D \& T o=A U D$ Accessed July 2020

31. Australian Bureau of Statistics. Australian statistical geography standard (ASGS): volume 5-remoteness structure, July 2016. 2018. Available at: https:// www.abs.gov.au/AUSSTATS/abs@.nsf/DetailsPage/1270.0.55.005July\%20 2016?OpenDocument. Accessed December 2018.

32. Zhen X, Lundborg CS, Sun X, Hu X, Dong H. Economic burden of antibiotic resistance in ESKAPE organisms: a systematic review. Antimicrob Resist Infect Control 2019; 8:137-60.

33. Founou RC, Founou LL, Essack SY. Clinical and economic impact of antibiotic resistance in developing countries: a systematic review and meta-analysis. PLOS One 2017; 12:e0189621.

34. Naylor NR, Pouwels KB, Hope R, et al. The health and cost burden of antibiotic resistant and susceptible Escherichia coli bacteraemia in the English hospital setting: a national retrospective cohort study. PLOS One 2019; 14:e221944.

35. Lambert ML, Suetens C, Savey A, et al. Clinical outcomes of health-careassociated infections and antimicrobial resistance in patients admitted to European intensive-care units: a cohort study. Lancet Infect Dis 2011 11:30-8.

36. Cheah AL, Spelman T, Liew D, et al. Enterococcal bacteraemia: factors influencing mortality, length of stay and costs of hospitalization. Clin Microbiol Infect 2013; 19:E181-9.

37. Beyersmann J, Wolkewitz M, Allignol A, Grambauer N, Schumacher M Application of multistate models in hospital epidemiology: advances and challenges. Biom J 2011; 53:332-50.

38. Morton A, Colson A, Leporowski A, Trett A, Bhatti T, Laxminarayan R. How should the value attributes of novel antibiotics be considered in reimbursement decision making? MDM Policy Pract 2019; 4:2381468319892237.

39. Thomas L, Bowen AC, Ly M, Connors C, Andrews R, Tong SYC. Burden of skin disease in two remote primary healthcare centres in northern and central Australia. Intern Med J 2019; 49:396-9.

40. Wozniak TM, Paterson D, Halton K. Review of the epidemiological data regarding antimicrobial resistance in Gram-negative bacteria in Australia. Infect Dis Health 2017; 22:210-8.

41. Davis JS, Jones CA, Cheng AC, Howden BP. Australia's response to the global threat of antimicrobial resistance: past, present and future. Med J Aust 2019; 211:106-108.e1.

42. Bowen AC, Daveson K, Anderson L, Tong SY. An urgent need for antimicrobial stewardship in indigenous rural and remote primary health care. Med J Aus 2019; 211:9-11.e1.

43. Brett JA, Johnson SA, Cameron DRM, et al. Carbapenemase-producing Enterobacteriaceae in Australian hospitals: outcome of point-prevalence screening in high-risk wards. J Hosp Infect 2019; 101:163-6.

44. Australian Institute of Health Welfare. Bloodstream infections associated with hos pital care 2017-18: Australian hospital statistics. Canberra, Australia: Australian Institute of Health Welfare, 2019. Available at: https://www.aihw.gov.au/reports/ health-care-quality-performance/bloodstream-infections-hospital-care-17-18 


\section{University Library}

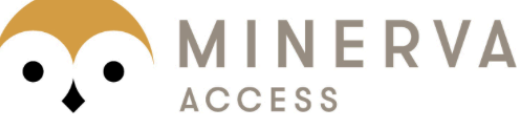

A gateway to Melbourne's research publications

Minerva Access is the Institutional Repository of The University of Melbourne

Author/s:

Lee, XJ;Stewardson, AJ;Worth, LJ;Graves, N;Wozniak, TM

Title:

Attributable Length of Stay, Mortality Risk, and Costs of Bacterial Health Care-Associated Infections in Australia: A Retrospective Case-cohort Study

Date:

2021-05-15

Citation:

Lee, X. J., Stewardson, A. J., Worth, L. J., Graves, N. \& Wozniak, T. M. (2021). Attributable Length of Stay, Mortality Risk, and Costs of Bacterial Health Care-Associated Infections in Australia: A Retrospective Case-cohort Study. CLINICAL INFECTIOUS DISEASES, 72 (10), pp.E506-E514. https://doi.org/10.1093/cid/ciaa1228.

Persistent Link:

http://hdl.handle.net/11343/252544

License:

CC BY-NC-ND 